\title{
Effect of Positive Events Recording Based on Positive Psychology on Healthy Behaviours and Readmission Rate of Patients After PCl: A Study Protocol for a Prospective, Randomized Controlled Trial
}

\author{
Yao yao Hu \\ Soochow University Medical College https://orcid.org/0000-0002-9088-6516 \\ Xin Jiang \\ Nanjing Medical University Affiliated Wuxi People's Hospital: Wuxi People's Hospital \\ Fang ying Mao \\ Soochow University Medical College \\ Jing Zhang \\ Soochow University Medical College \\ Lin Liu \\ First Affiliated Hospital of Soochow University Department of Cardiology \\ Jie Gu \\ First Affiliated Hospital of Soochow University Department of Cardiology \\ Qing Wu (D329349583@qq.com) \\ First Affiliated Hospital of Soochow University Department of Cardiology \\ Yin Wu \\ First Affiliated Hospital of Soochow University Department of Cardiology
}

Study protocol

Keywords: Positive, Coronary heart disease, Behaviours, Readmission rate, Randomized controlled trial

Posted Date: June 29th, 2021

DOI: https://doi.org/10.21203/rs.3.rs-654231/v1

License: @ (1) This work is licensed under a Creative Commons Attribution 4.0 International License. Read Full License 


\section{Abstract}

Background: Unhealthy behaviours of coronary heart disease (CHD) patients are closely related to the occurrence of major heart events, which increases the readmission rate and brings heavy economic burden to families and society. Therefore, it is necessary for health care workers to take active preventive and therapeutic measures to keep or establish healthy behaviours of patients. Positive psychological intervention has been proved to be effective, but it has not been reported in the field of $\mathrm{CHD}$ in China. The purpose of this study is to explore the effects of positive events recording based on positive psychology on the healthy behaviours, readmission rate and anxiety of patients with CHD, in order to provide new ideas for the development of secondary prevention strategies for CHD.

Methods: This is a prospective, single-center, randomized controlled trial (RCT). The subjects will be enrolled from the Department of Cardiology, the First Affiliated Hospital of Soochow University. There are 80 cases in total, according to the random number table, the subjects are randomly divided into the intervention group $(n=40)$ and the control group $(n=40)$. The patients in the intervention group will receive the intervention of recording positive events once a week for 3 months, while the patients in the control group receive conventional nursing, and each group will be followed up for 6 months. The primary outcomes will include healthy behaviours, readmission rate and anxiety, the secondary outcomes will include psychological capital, subjective well-being and corresponding clinical laboratory indicators. The protocol was approved by the Medical Ethics Committee of Soochow University (approval No. SUDA20200604H01) and is performed in strict accordance with the Declaration of Helsinki formulated by the World Medical Association. All participants provide written informed consent.

Discussion: This study will verify whether positive events recording based on positive psychology can make patients maintain healthy behaviours, reduce readmission rate and improve anxiety after $\mathrm{PCI}$. Then, this study will provide new ideas and references for the development of secondary prevention strategies for patients with CHD.

Trial registration: Chinese Clinical Trials Registry, 2000034538. Registered on 10 July 2020.

\section{Background}

Coronary heart disease (CHD) is an acute myocardial ischemia and hypoxia disease caused by coronary artery stenosis, which is one of the highest mortality diseases in the world[1], and the mortality rate is the third in China[2]. With the increasing incidence rate and mortality of $\mathrm{CHD}$ [3], has resulted in a large amount of medical expenses, making coronary heart disease one of the main reasons for the economic burden of chronic non-communicable diseases in China[4]. At present, percutaneous coronary intervention $(\mathrm{PCl})$ has become one of the main methods for treating CHD because of its characteristics such as low trauma, fast recovery and short hospitalization time. Although $\mathrm{PCl}$ significantly reduces the symptoms of CHD, reduces mortality, and significantly improves quality of life[5], there is still a risk of major adverse cardiovascular events (MACE) in some patients, which can seriously affect prognosis and increase readmission rates[67].

The prognosis of patients with CHD is determined by various factors. Studies have shown that unhealthy behaviours increased the risk of in-stent restenosis (ISR) and the occurrence of MACE [7-8]. In addition, negative psychology also affected the prognosis of patients after PCl. Iles-Smith et al.[9] confirmed that anxiety was a predictor of readmission. Frasure-Smith et al.[10] concluded that mood disorders were associated with the occurrence of MACE within 2 years in patients with coronary heart disease. However, one study has proved that emotional intervention in elderly patients with heart failure can help them improve heart function, reduce the occurrence of falls, and improve the quality of life[11]. It has also been found that psychological interventions for patients can improve treatment cooperation and can also help patients change their lifestyle and establish healthy behaviours [12-14]. Therefore, patients will benefit most if all known risk factors are controlled. 
Positive psychology is a part of psychology, which mainly helps people to form good psychological quality and behaviour patterns, focusing on positive subjective experience, positive personality traits and positive social environment, and fully exploiting the individual's own strength[15-16]. At present, positive psychology has been widely used in many fields abroad, such as obstetrics and gynecology, diabetes, etc. Corno et al.[17] found that positive psychological intervention can help pregnant women reduce anxiety and depression and improve happiness index. Huffman et al.[18] carried out positive psychological intervention on patients with type 2 diabetes and found that it can help patients improve treatment compliance, also Hoeppner et al.[19] showed that positive psychological intervention can help people quit smoking. However, the development of positive psychology in China is relatively less, mainly in the fields of education[20], mental illness[21], etc. The study by Lan et al.[22] clarified that psychological intervention based on positive psychology can help patients maintain a good psychological state, improve depression and improve self-care ability. Zhu et al.[23] also confirmed that positive psychological intervention can improve the disability acceptance and self-care ability of stroke inpatients.

At present, positive psychological has been implemented in a variety of ways, including expressive writing, mindfulness therapy, gratitude therapy, hope therapy, etc. These methods have a positive effect on reducing negative emotions and improving subjective well-being of patients. Writing is a unique human behaviour of information expression, communication and creation, and the positive events recording in this study is to express the positive experience or feelings related to the individual through writing[24], which has also been applied to relevant fields and achieved certain curative effects. For example, an intervention study of 411 participants, conducted by Seligeman et al.[25]showed that recording positive events significantly improved the well-being of those who were involved. Burton et al.[26] found that recording positive events can help people improve their cognition. Suhr et al.[27] has shown that the benefits of writing positive events to help people with mental illness stabilize their mental health. Chinese scholar Xu et al.[28] found that writing positive events and expressing can effectively improve (human immunodeficiency virus)HIV/(acquired immune deficiency syndrome)AIDS patients' self-discrimination, negative emotions and improve self-control level, Shang[29] also helped patients relieve negative psychology, enhance positive psychology, promote positive coping and improve the quality of life by giving the same intervention to patients with inflammatory bowel disease. However, there are few reports about positive events recorded in patients after $\mathrm{PCl}$ in China, and most of the positive psychological intervention programs in China have poor repeatability and lack of systematic and targeted intervention programs. In view of the simple operation of positive event recording, the time is short, economical and convenient, and the effect is remarkable, it is necessary to carry out positive psychological intervention for patients after $\mathrm{PCl}$ in China to explore its intervention effect and formulate a more suitable positive psychological intervention program for patients with $\mathrm{CHD}$ in China.

The purpose of this study is to explore whether the implementation of positive events recording has an impact on the healthy behaviours, readmission rate and anxiety of patients after $\mathrm{PCl}$ in the theoretical framework of positive psychology, in order to provide reference for improving the quality of specialized nursing and formulating secondary prevention strategies for patients with CHD.

\section{Objectives}

The general aim of this study is to evaluate the efficacy of positive events recording in patients after PCl.

The primary objective is to explore whether recording positive events will promote or maintain healthy behaviours, reduce readmission rate and improve anxiety of patients after $\mathrm{PCl}$.

The secondary objective is to explore whether recording positive events can promote the positive emotions, subjective well-being and clinical laboratory indicators (such as total cholesterol (TC), triglyceride (TG), high-density lipoprotein (HDL), low-density lipoprotein (LDL), height and weight, etc.) of patients after PCI. 


\section{Methods}

\section{Study design and setting}

This is a randomized controlled trial, which will be conducted in the Department of Cardiology, the First Affiliated Hospital of Soochow University. A written informed consent will be obtained from each individual by the researcher after the individual has received a sufficient explanation and period of time in which to make a thoughtful decision. All the patients will be randomized 1:1 into the intervention group $(n=40)$ or the control group $(n=40)$. The intervention group is given 3-month positive events recording intervention on the basis of conventional nursing, while the control group received conventional nursing, and the two groups will be followed up for 6 months. The Cardiovascular Medicine Department of the First Affiliated Hospital of Soochow University is currently the largest cardiovascular disease diagnosis and treatment center in South Jiangsu Province, China. It has 180 beds, four cardiac catheterization rooms with advanced equipment, as well as a cardiac supersonic room and cardiac function room. Annually, an average of 5000 open-heart surgery candidates and 400 emergency operations are treated in the department. The protocol design is based on the Consolidated Standards of Reporting Trials (CONSORT) guidelines and Standard Protocol Items:

Recommendations for Interventional Trials (SPIRIT) checklist (see Additional file 3). Figure 1 presents a schematic flow diagram of the study.

\section{Participants}

\section{Inclusion Criteria}

Participants must meet the following criteria:

1. those who are aged 8 years or older

2. those who meet the diagnostic criteria of coronary heart disease in the ninth edition of Internal Medicine, including stable angina, unstable angina, myocardial infarction and acute coronary syndrome [30]

3. those who after $\mathrm{PCl}$

4. those who agree to participate and provide written informed consent

5. those who have been living in Suzhou for a long time or are not leaving Suzhou for half a year

6. those who are clear-headed, normal-thinking and able to communicate with each other

\section{Exclusion Criteria}

Applicants who meet any of these conditions cannot participate in this study:

1. those who have severe cognitive impairment, mental illness and non-cooperation

2. those who have other serious diseases that affect survival, such as advanced cancer

3. those who could not record for 4 consecutive weeks

4. other reasons determined by the investigators that make participation in the clinical trial inappropriate

\section{Withdrawal criteria}

Participants who meet the criteria summarized below are withdrawn from the study. The subjects who are withdrawn after randomization will be followed up for outcomes. Reasons for withdrawal will be documented in follow-up records and data will be analyzed using the intention-to-treat (ITT) principle.

1. all subjects have the right to withdraw for any reason 
2. those who could not complete the test as required, with serious adverse events and complications

3. failed to have three consecutive telephone connections during the follow-up period

4. at the end of the study

\section{Randomization}

Eligible participants who will provide written consent to take part in clinical trial will be randomly assigned to an intervention group or a control group with an allocation ratio of 1:1. Random sequencing will be generated by an independent professional statistician using the SPSS 25.0 software to generate 80 random numbers. The serial numbers assigned to each patient will be placed in an opaque sealed envelope and kept by the personnel not involved in the specific study. The participants will be randomly assigned to two groups, and each group will have 40 individuals.

\section{Blinding}

Member of the research team who enters the information is the only ones who know about the participantsc allocation. The data collectors, random allocation program makers, data analysts and outcome evaluators are blinded to group allocation. And the participants are similarly blinded to their own group allocation. Participants are similarly blinded to their own group allocation and they will be told not to share their positive events records with others. Results from the outcome measures will not be revealed to the participants until after all recruitment, treatment, and assessments have been performed for all 80 participants.

\section{Procedures}

\section{Recruitment}

Participants will be recruited from The First Affiliated Hospital of Soochow University located in Suzhou, China. This institution will advertise the trial by posting recruitment posters on the hospital website or bulletin board.

\section{Study schedule}

The items to be measured at each visit are presented in Table1.

\section{Baseline assessment}

After the screening visit, if a participant fulfills the inclusion criteria and has signed the informed consent form, he will be assigned to the baseline assessment. The base-line assessment takes place at visit 1. Baseline assessment includes patientsç basic information (such as age, gender, education, income, occupation, medical insurance, comorbidity, exercise, smoking habit, drinking habit, sleep pattern, etc.), body measurement (height, body weight, body mass index(BMI), blood pressure(BP), heart rate, etc.), clinical indicators (TG, TC, LDL, and blood glucose, etc.), questionnaires (Health Promotion Lifestyle Scale II (HPLP II), Self-rating Anxiety Scale (SAS), Positive Psychological Capital Questionnaire, General Well-Being Scale).

\section{Table 1 Study schedule for the trial}




\begin{tabular}{|c|c|c|c|c|c|c|c|c|c|}
\hline \multirow[t]{2}{*}{ Items } & \multirow{2}{*}{$\begin{array}{l}\text { Screening } \\
\text { Visit } 1 \\
\text { (day-7 to } \\
-2 \text { ) }\end{array}$} & \multicolumn{7}{|c|}{ Post allocation } & \multirow{2}{*}{$\begin{array}{l}\text { End of } \\
\text { treatment } \\
\text { visiting } 3 \\
\text { months } \\
\text { Visit9 } \\
\text { (week24) }\end{array}$} \\
\hline & & $\begin{array}{l}\text { Visit } \\
2 \\
\text { (day } \\
0)\end{array}$ & $\begin{array}{l}\text { Visit } 3 \\
\text { (week1) }\end{array}$ & $\begin{array}{l}\text { Visit } \\
4 \\
\text { (week } \\
\text { 2) }\end{array}$ & $\begin{array}{l}\text { Visit } \\
5 \\
\text { (week } \\
3 \text { ) }\end{array}$ & $\begin{array}{l}\text { Visit } 6 \\
\text { (week4) }\end{array}$ & $\begin{array}{l}\text { Visit } \\
7 \\
\text { (week } \\
\text { 8) }\end{array}$ & $\begin{array}{l}\text { Visit8 } \\
\text { (week12) }\end{array}$ & \\
\hline Informed consent & $x$ & & & & & & & & \\
\hline $\begin{array}{l}\text { Inclusion/exclusion } \\
\text { criteria }\end{array}$ & $x$ & & & & & & & & \\
\hline Randomization & & $x$ & & & & & & & \\
\hline Intervention & & $x$ & $x$ & $x$ & $x$ & $x$ & $x$ & $x$ & $x$ \\
\hline Basic information $^{a}$ & $x$ & & & & & & & & \\
\hline $\begin{array}{l}\text { Body } \\
\text { measurement }^{b}\end{array}$ & $x$ & $x$ & $x$ & $x$ & $x$ & $x$ & $x$ & $x$ & $x$ \\
\hline Vital signs & $x$ & $x$ & $x$ & $x$ & $x$ & $x$ & $x$ & $x$ & $x$ \\
\hline Medical history ${ }^{c}$ & $x$ & & & & & & & & \\
\hline $\begin{array}{l}\text { General physical } \\
\text { examination }\end{array}$ & $x$ & & & & & & & $x$ & $x$ \\
\hline $\begin{array}{l}\text { Compliance } \\
\text { monitoring }\end{array}$ & & & & $x$ & & & & $x$ & \\
\hline Healthy behaviours & $x$ & & & & & & & $x$ & $x$ \\
\hline Readmission rate & & & & & & & & $x$ & $x$ \\
\hline Anxiety & $x$ & & & & & & & $x$ & $x$ \\
\hline $\begin{array}{l}\text { Psychological } \\
\text { capital }\end{array}$ & $x$ & & & & & & & $x$ & $x$ \\
\hline $\begin{array}{l}\text { Subjective well- } \\
\text { being }\end{array}$ & $x$ & & & & & & & $x$ & $x$ \\
\hline Clinical indicators $^{d}$ & $x$ & & & & & & & $x$ & $x$ \\
\hline $\begin{array}{l}\text { Adverse event } \\
\text { monitoring }\end{array}$ & & & $x$ & $x$ & $x$ & $x$ & $x$ & $x$ & $x$ \\
\hline
\end{tabular}

${ }^{a} A g e$, gender, job, medical insurance, exercise, smoking habit, drinking habit, sleep pattern, etc.

${ }^{b}$ Height and weight, but only weight for visit 2 and follow-up

CIncluding general medical history and family history of CHD

dIncluding TG, TC, LDL, HDL, blood glucose etc.

Intervention 
After randomization, the patients in the control group will receive conventional nursing, and the patients in the intervention group will record positive events for 3 months in addition to conventional nursing, that is, once a week for 12 weeks. Each patient in the intervention group will be assigned a notebook to record positive events and a health education manual. The specific implementation plan is as follows:

\section{Intervention group}

On the basis of the same conventional nursing as the control group, the following measures were given:

1. Follow up: mainly by phone or Wechat, once a week in the first month after discharge, and once a month after that until 3 months. Each follow-up time is about 5-10 minutes. If the patient has any questions, the time can be extended as appropriate.

2. Positive events recording guidance: the intervention plan was designed by our team based on the positive psychology intervention program by Seligman[25] and also based on a review of literature and expert consultation[31-33]. Related literature reported that the period of positive psychological intervention is generally more than 8 weeks[34]. Shang[29] improved the patientsç negative emotions and promoted positive psychology through 8-week positive events recording intervention. Lan[22] conducted 10-week intervention on type 2 diabetes patients, and their depression and anxiety psychology was also effectively improved, and their self-care ability was improved too. So, the implementation time of positive events recording in this study is planned to be 3 months (see table 2).

3. Recording log: each individual is given a recording log and asked to record the number and time of recording every day. The data will be used to assess intervention compliance.

\section{Table 2 Outline of positive events recording for patients after PCl}

\begin{tabular}{|ll|}
\hline Week & Specific measures \\
\hline 1 & $\begin{array}{l}\text { Review } 3 \text { events about your positive state this week and record them (for example, I finished my exercise } \\
\text { today, and I took medicine under the doctor's advice today etc. It has nothing to do with the size of the } \\
\text { matter) }\end{array}$ \\
\hline 2 & Evaluate your strengths and apply them \\
\hline 3 & Write a thank-you note \\
\hline 5 & Complete 3 enjoyable and meaningful activities on your own \\
\hline 6 & Review previous successful experiences \\
\hline 7 & Eliminate negativity (When there are negative emotions, record one thing that makes you happy) \\
\hline 8 & Develop your own interests and hobbies \\
\hline 9 & Set realistic goals and achieve them \\
\hline 10 & Review the good events from your past life and summarize your life \\
\hline $11-$ & For the next two weeks, repeat the above and practice or write it down \\
\hline 12 &
\end{tabular}

\section{Control group}


1. Conventional nursing: on the first day after enrollment, researchers communicate with patients one-on-one, explain $\mathrm{CHD}$ related knowledge to patients, including risk factors, diet strategies, weight control, physical exercise, blood pressure and lipid management, etc. After the completion of the education, give the patients the corresponding education manual.

2. Follow up by phone or Wechat: once per month for 3 months. Each call lasting about 5-10 min, receiving telephone or Wechat counseling from patients.

\section{Outcome}

The primary outcome indicators are healthy behaviours, readmission rate and anxiety level, the secondary outcome indicators are psychological capital, subjective well-being, clinical indicators (including TC, LDL, HDL, blood glucose, body mass index, etc.).

\section{Primary outcome measure}

1. Healthy behaviours: The Health Promotion Lifestyle profile II (HPLP II) will be used to measure patientçs healthy behaviours. The scale was revised by American nursing scientist Walker[35], which mainly includes 6 dimensions and 52 items. The total Cronbach's a coefficient of the scale is 0.94 , and the Cronbach's a number of each dimension is $0.79-0.87$. Each item is scored by grade $1-4$, the higher the score, the better the healthful lifestyle. It will be checked at visit 1,8 and 9 .

2. Readmission rate: In this study, readmission is defined as the readmission caused by cardiovascular problems, including hypertension, angina, arrhythmia, cardiac insufficiency, etc., excluding the cases of readmission due to cold, tumor and other factors. The readmission will be followed up by telephone for 3 months. The readmission information will be collected at visit 8 and 9.

3. Anxiety: The self-rating anxiety scale (SAS) will be used to measure the anxiety of patients with CHD. The scale was compiled by Chinese professor Zung (1971), there are 20 items on the scale, each item is scored by grade 1-4, and items 5, 9, 13, 17 and 19 are scored in reverse. According to the results of Chinese norm model results[36], the cutoff value of the SAS standard score is 50, of which 50-59 is mild anxiety, 60-69 is moderate anxiety, and over 69 is severe anxiety. The scale has been widely used in China and its validity and reliability have been verified (Cronbach's a 0.82). The anxiety score will be measured at visit1, 8 and 9.

\section{Secondary outcome measure}

1. Psychological capital: The positive psychological capital questionnaire (PPQ) is used to evaluate the patientçs positive psychology. The questionnaire was revised by Professor Zhang et al.[37] of Nankai University on the positive psychological capital questionnaire compiled by Lutas et al. The questionnaire includes 26 items, Cronbach's a coefficient is 0.92 , Cronbach's a coefficient of each dimension is $0.81,0.76,0.75,0.78$ respectively. The higher the score, the better the psychological capital. It will be measured at visit1, 8 and 9 .

2. Subjective well-being: The general well-being scale (GWB) is mainly used to evaluate the subjective well-being of patients. The scale was amended by Duan[38], a Chinese scholar, on the basis of Fazio. The scale includes 18 items, and the higher the score, the stronger the subjective well-being. The internal consistency coefficient of the scale is 0.91 for males and 0.95 for females. The internal consistency coefficient of retest is 0.85 , and the correlation coefficient between subscale and total scale is $0.56-0.88$. The subjective well-being score will be measured at visit1, 8 and 9.

3. Clinical indicators: Clinical indicators will be followed up by researchers through Wechat or telephone to collect relevant clinical laboratory indicators of patients in 3 months, including BP, blood lipid, blood glucose, BMI, etc. The Clinical indicators will be collected at visit1, 8 and 9. 


\section{Safety outcome measure}

The safety assessment will be performed for all subjects who have been randomized and recorded positive events more than once. The subjectsç vital signs and general physical status will be examined at every visit. All participants will be required to report any adverse events $(A E)$ that occur during the trial of visits $2,3,4,5,6,7,8$ and 9 . All $A E s$ that occur after the start of this trial should be recorded in the case report form, regardless of whether they are related to positive events recording or not. All AEs will be evaluated for causality.

\section{Compliance monitoring and management}

The researchers will collect the recording logs of the patients after 2 weeks of positive events recording for the purpose of calculating compliance. The compliance rate $(\%)=$ [continuous recorder as required/ total number of interventions] * $100 \%$. Only when the compliance is $\geq 70 \%$, the research will continue, otherwise, find out the reasons and carry out scheme rectification.

\section{Sample size calculation}

Calculated according to the formula of sample size estimation based on the comparison of the mean of two independent samples [39]: ${ }^{n_{1}=n_{2}=2\left[\left(Z_{\alpha}+Z_{\beta}\right) \sigma / \delta\right]^{2}+\frac{1}{4} Z^{2} \alpha_{\beta}} \sigma$ is the standard deviation, $d$ is the difference between the two population means, $n_{1}$ and $n_{2}$ are the required contents of the two samples respectively, $Z_{a}$ and $Z_{\beta}$ are $Z$ values corresponding to the type I error probability and the type II error probability respectively. At bilateral $a=0.05$, power $=0.8$, $Z_{a / 2, v}=1.96, Z_{\beta, v}=0.842, \sigma=12.68, \delta=10.39$, the sample size for each group is 25 cases. Considering the $20 \%$ sample loss rate and other factors, the total number of samples is expected to be 80 .

\section{Data management and quality control of data}

Both the case record form (CRF) and web-based electronic database will be used to manage individual participant data. To protect confidentiality, the files are stored in a secure and locked place and manner, the subject identification and private information will be deleted from all study documents. Quality control of the data will be handled at two different levels: the investigators will be required to ensure the accuracy of the data as the first level of control when they input the records in CRF. The second level will include data monitoring and validation that will be carried out by two full-time graduate students who will not be involved in the intervention or data collection. All the data will be double-inputted into the computer using Epidata3.0 software. After finishing the data entry and dealing with the query, the database will be locked under the orders of the principal investigator, and the SPSS software will be used for data analysis. No one is allowed to view the database without authorization, otherwise, they should notify the principal investigator.

\section{Statistical analysis}

The Kolmogorov-Smirnov test will be used to test the normal distribution of continuous variables. Continuous variables will be presented by mean \pm standard deviation (SD) if they are normally distributed or by median with interquartile range if they are not normally distributed. For the comparison of measurement data between the two groups, t-test of two independent samples will be used for normal distribution data, and Mann Whitney U rank sum test will be used for nonnormal distribution data. The comparison of count data between the two groups will be performed by $c^{2}$ test or Fisherçs exact test. An independent statistics expert will perform statistical analysis in a blind manner. SPSS for windows version 25.0 (SPSS Inc., Chicago, IL, USA) will be used for statistical analysis. The statistical level of significance will be set at $P<0.05$. 


\section{Discussion}

At present, CHD has become the main cause of death of urban and rural residents in China[3], and is also one of the main reasons for the economic burden of chronic noncommunicable diseases[4]. Its occurrence and development are closely related to unhealthy behaviours such as unreasonable diet, smoking, drinking and so on[40]. Only relying on drugs or surgical treatment will not change the pathological progress of coronary atherosclerosis. Studies have shown that the higher incidence of ISR and MACE are associated with unhealthy behaviours[7-8]. Therefore, it is of profound significance to change the unhealthy behaviours of patients to reduce the occurrence of cardiovascular events.

The American college of cardiology (ACC) and the American heart association (AHA) issued the "2019 cardiovascular primary prevention guidelines", which pointed out that adhering to a healthy lifestyle is an important measure to prevent cardiovascular disease[41]. Among the 15 special activities of healthy China, measures to promote healthy lifestyle, such as reasonable diet, tobacco control and national exercise, have been put in a prominent position. These above show the importance of healthy behaviours to the organism. Therefore, it is necessary to help patients establish healthy behaviours.

According to the "extension-construction" theory proposed by Fredrickson[42], this theory believes that positive emotions can expand the thinking and action ability, and then build a series of enduring resources of individuals, so as to promote health and development. This study is based on positive psychology of the experimental design, paying more attention to the potential positive forces of patients, helping patients to tap their own good quality, improve positive emotions, and promote positive development. Many experimental studies have confirmed that positive psychological intervention can help patients to improve anxiety, depression and other bad psychology, improve their quality of life, improve treatment compliance, help people quit smoking[17-19]. This study applies positive psychological intervention to patients after $\mathrm{PCl}$ for the first time, through systematic practice and training, to determine whether positive event records based on positive psychology will promote the establishment or maintenance of healthy behaviours, reduce the rate of readmission, improve negative emotions such as anxiety and depression, improve subjective well-being, etc. Which will provide new ideas and references for the formulation of secondary prevention strategies for CHD.

Our trial has several advantages. Firstly, the purpose of our study is to explore the effects of positive events recording on healthy behaviours, readmission rate and negative emotions such as anxiety and depression etc. At home and abroad, there have been studies on the clinical application of writing positive events, and our research team has previously conducted cross-sectional surveys of patient's healthy behaviours, readmission status, and negative emotions such as anxiety and depression after $\mathrm{PCl}$, which has a solid practical and theoretical foundation. Secondly, the intervention measure of this study is to record positive events by patients themselves, which can mobilize the enthusiasm of patients to actively participate in disease management. Thirdly, positive events recording discussed in this study belong to the secondary preventive measures for patients with CHD, which is also in line with the "two heart" nursing mode advocated by China now. This form of nursing intervention research is rarely reported in the field of CHD in China.

Despite the advantages of its results, our study has the following limitations. First of all, due to limited time, manpower and funding, this study is only conducted in a hospital in Suzhou. In future trials, more patients with PCl in different levels of hospitals should be included to explore the role of positive psychology. Secondly, only one positive psychological intervention therapy is used in this study. In the future, a variety of progressive positive psychological intervention methods can be combined to test to determine a better treatment plan.

After the completion of this study, we will conduct large-sample, multi-center and high-quality RCTs in future studies to further verify the role of positive events recording in the maintenance and promotion of health, and provide reliable evidence-based basis for the clinical promotion and application of positive events recording.

Page $10 / 15$ 


\section{Trial status}

The study was registered with Chinese Clinical Trial Registry (registration number: ChiCTR2000034538) on 10 July 2020. This study started to recruit volunteers on 11 July 2020, and is expected to complete the recruitment by 31 June 2021. So far, 20 volunteers have been recruited for this trial.

\section{Abbreviations}

ACC: American college of cardiology; AE: Adverse events; AHA: American heart association; AIDS: Acquired immune deficiency syndrome; BMI $\triangle$ Body mass index; BP: Blood pressure; CHD: Coronary heart disease; CRF: Case record form; GWB: General well-being scale; HDL: High density lipoprotein; HIV: Human immunodeficiency virus; HPLP: Healthpromotion lifestyle profile; ISR: In-stent restenosis; ITT: Intention-to-treat; LDL: Low density lipoprotein; MACE: Major adverse cardiovascular events; PCI区Percutaneous coronary intervention; PPQ: Positive psychological capital questionnaire; RCT: Randomized controlled trial; SAS: Self-rating anxiety scale; SD: Standard deviation; TC: Total cholesterol; TG: Triglyceride.

\section{Declarations}

\section{Acknowledgements}

This study is supported by Suzhou science and technology bureau (grant no.sys2018018). We thank everyone who helped us in the development of this study protocol.

\section{Funding}

This trial is supported by Suzhou Science and Technology Bureau (grant no.sys2018018). The protocol, case report form development, and reporting of the study will be conducted independently by the study investigators. The funding body did not have any role in the study design, data collection, or the writing of the manuscript, or decision to submit the manuscript for publication.

\section{Authorsç contributions}

YYH and QW wrote the first manuscript for this trial. LL, XJ, JG and $Y W$ designed the study, coordinated the acquisition of the data, and helped to draft the manuscript. YYH and QW calculated the sample size and determined the methods of statistical analysis. QW will monitor this trial. YYH, QW, XJ, LL, YW, JZ and FYM participated in the design of the study. QW critically revised the manuscript. All authors read and approved the manuscript.

\section{Competing interests}

The authors declare that they have no competing interests.

\section{Consent for publication}

Not applicable.

\section{Availability of data and materials}

The results of the trial will be disseminated through scientific journals or presentations at scientific conferences. So far, the public access to any trial data set is still unplanned. 
This study had been approved by the Medical Ethics Committee of Soochow University (approval No.

SUDA20200604H01) (Additional file 1) and conforms to the principles of the Helsinki declaration. The present trial is registered at http://www.chictr.org.cn (ChiCTR2000034538). All participants will sign the informed consent form (these are available from the corresponding author) (Additional file 2) before participation.

\section{Author details}

${ }^{1}$ Department of Cardiology, the First Affiliated Hospital of Soochow University, Suzhou, China

${ }^{2}$ Nursing Department, the Affiliated Wuxi Peopleçs Hospital of Nanjing Medical University, Wuxi, China.

${ }^{3}$ School of nursing, Soochow University, Suzhou, China.

\section{References}

1. GBD 2016 Causes of Death Collaborators. Global, regional, and national age-sex specific mortality for 264 causes of death, 1980-2016: a systematic analysis for the Global Burden of Disease Study 2016. Lancet. 2017;390(10100):1151-1210.

2. Yao JP, Wu Y, Chen K. Internal medicine nursing. 2th ed. Beijing: Peking University Medical Press; 2015.

3. Hu SS, Gao RL, Liu LS, Zhu ML, Wang W, Wang YJ, et al. Summary of the 2018 report on cardiovascular diseases in China. Chinese Circulation Journal. 2019;34(03):209-220.

4. Hu JP, Rao KQ, Qian JC, Wu J. The study of economic burden of chronic non-communicable diseases in China. Chin J Prey Contr Chron Non-comrmm Dis. 2007;15(3):189-193.

5. Head SJ, Milojevic M, Daemen J, Ahn JM, Boersma E, Christiansen EH, et al. Mortality after coronary artery bypass grafting versus percutaneous coronary intervention with stenting for coronary artery disease: a pooled analysis of individual patient data. Lancet. 2018;391(10124): 939-948.

6. Rao SV, Kaltenbach LA, Weintraub WS, Roe MT, Brindis RG, Rumsfeld JS, et al. Prevalence and outcomes of sameday discharge after elective percutaneous coronary intervention among older patients. Jama.2011;306(13):14611467.

7. Liu B, Tao GZ. Analysis of related factors of stent restenosis after coronary stent implantation. Chinese Circulation Journal. 2017;32(z1):92.

8. Xu L, Qu YP, Li LH. Relationship between unhealthy behaviors and major adverse cardiac events in patients with coronary heart disease. Chin J Mod Nurs.2019;25(24):3133-3137.

9. Iles-Smith H, McGowan L, Campbell M, Mercer C, Deaton C. A prospective cohort study investigating readmission, symptom attribution and psychological health within six months of primary percutaneous coronary intervention. Eur J Cardiovasc Nurs. 2015;14(6):506-515.

10. Frasure-Smith N, Lespérance F. Depression and anxiety as predictors of 2-year cardiac events in patients with stable coronary artery disease. Arch Gen Psychiatry. 2008;65(1):62-71.

11. Cao XC, Huang GR, Liu X, Duan MQ, Xu XJ, Wang XH. Impact of comprehensive geriatric assessment on treatment outcomes of chronic heart failure in elderly patients complicated with emotional disorders. Chin J Geriatr. 2019;38(9):976-979.

12. Shepardson RL, Buchholz LJ, Weisberg RB, Funderburk JS. Psychological interventions for anxiety in adult primary care patients: A review and recommendations for future research. J Anxiety Disord. 2018; 54:71-86.

13. Wide U, Hagman J, Werner H, Hakeberg M. Can a brief psychological intervention improve oral health behaviour? A randomised controlled trial. BMC Oral Health. 2018;18(1):163. 
14. Gate L, Warren-Gash C, Clarke A, Bartley A, Fowler E, Semple E, et al. Promoting lifestyle behaviour change and wellbeing in hospital patients: a pilot study of an evidence-based psychological intervention. J Public Health (Oxf). 2016;38(3): e292-e300.

15. Seligman ME, Csikszentmihalyi M. Positive psychology. An introduction. Am Psychol. 2000;55(1):5-14.

16. Xin XL, Sun HY. Progress of research on the application of positive psychology in nursing education in China. Chin $\mathrm{J}$ Nurs Educ. 2017;14(9):711-714.

17. Corno G, Etchemendy E, Espinoza M, Herrero R, Molinari G, Carrillo A, et al. Effect of a web-based positive psychology intervention on prenatal well-being: A case series study. Women Birth. 2018;31(1): e1-e8.

18. Huffman JC, DuBois CM, Millstein RA, Celano CM, Wexler D. Positive psychological interventions for patients with type 2 diabetes: rationale, theoretical model, and intervention Development. J Diabetes Res. 2015: 428-349.

19. Hoeppner BB, Hoeppner SS, Carlon HA, Perez GK, Helmuth E, Kahler CW, et al. Leveraging positive psychology to support smoking cessation in nondaily smokers using a smartphone app: feasibility and acceptability Study. JMIR Mhealth Uhealth. 2019;7(7): e13436.

20. Yan $M, W u$ YZ. The intervention effect of directed group counseling on college students' self-acceptance. Chin J Sch Health. 2020; 41(2):298-301.

21. Liu XL, Xiong YE, Yu GL, Li MZ. Research on improvement of the mental health of the elderly shidu person under positive psychological view. Chongqing Medicine. 2020,49(12):2009-2012.

22. Lan Y, Liu WW, Zhou XR, Lu LY, Liu SQ, Pang T. The application of psychological intervention based on positive psychology theory in patients with type 2 diabetes with depression. Int J Nurs. 2019;38(22):3683-3687.

23. Zhu T, Huang YL, Fang YC, Liu AH, Luo XL, Xu JM. Effect of positive psychological intervention based on PERMA model on disability acceptance and self-care disability in stroke patients. Chinese Nursing Research.2020;34(06):965-970.

24. Wang Y, Wang ZH. Expressive writing and its role to improve physical and mental health. Advances in Psychological Science. 2010,18(02):314-321.

25. Seligman ME, Steen TA, Park N, Peterson C. Positive psychology progress: empirical validation of interventions. Am Psychol. 2005;60(5):410-421.

26. Burton CM, King LA. The health benefits of writing about positive experiences: the role of broadened cognition. Psychol Health. 2009;24(8):867-79.

27. Suhr M, Risch A K, Wilz G. Maintaining mental health through positive writing: Effects of a resource diary on depression and emotion regulation. J Clin Psychol.2017;73(12):1586-1598.

28. Xu XH, Fu Y, Song B, Xu HX, Mian Y. Effectiveness of expressive writing in reducing self-stigma among homosexual men living with HIV/AIDS. Nurs J Chin PLA. 2019;36(8):1-5.

29. Shang XC. Effect of positive psychological intervention based on written expression on psychological status of patients with inflammatory bowel disease. Nanjing Medical University.2019.

30. Ge JB, Xu YJ. Internal Medicine. 9th Edition. Beijing: People's Medical Publishing House;2018.

31. Huffman JC, Albanese AM, Campbell KA, Celano CM, Millstein RA, Mastromauro CA, et al. The positive emotions after acute coronary events behavioral health intervention: design, rationale, and preliminary feasibility of a factorial design study. Clin Trials. 2017;14(2):128-139.

32. Celano CM, Albanese AM, Millstein RA, Mastromauro CA, Chung WJ, Campbell KA, et al. Optimizing a positive psychology intervention to promote health behaviors following an acute coronary syndrome: The Positive Emotions after Acute Coronary Events-III (PEACEIII) randomized factorial trial囚Psychosom Med.2018;80(6):526-534.

33. Mongrain M, Anselmo-Matthews T. Do positive psychology exercises work? A replication of Seligman et al. (2005). J Clin Psychol.2012;68(4):382-389. 
34. Bolier L, Haverman M, Westerhof GJ, Riper H, Smit F, Bohlmeijer E. Positive psychology interventions: a metaanalysis of randomized controlled studies. BMC Public Health. 2013; 13:119.

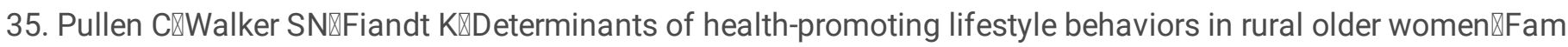
Community Health. 2001;24(2)『49-72】

36. Dai XY. Handbook of commonly used psychological assessment scales (Revised Edition). Beijing: People's military medical publishing house; 2015.

37. Zhang K, Zhang S, Dong YH. Positive psychological capital: measurement and relationship with mental health. Studies of Psychology and Behavior. 2010;8(01):58-64.

38. Duan JH. Experimental results and Analysis of total Well-being Scale in Chinese college Students. Chinese Journal of Clinical Psychology.1996(01):56-57.

39. Zhang MZ, Li HM, Lv DB. Applied medical statistics and SAS application. Suzhou University Press;2015.

40. Wang G, Zhao L, Li TC. Clinical characteristics and risk factors of elderly male and female CHD patients. Chin J Geriatr Heart Brain Vessel Dis. 2019;21(5):483-486.

41. Arnett DK, Blumenthal RS, Albert MA, Buroker AB, Goldberger ZD, Hahn EJ, et al. 2019 ACC/AHA Guideline on the Primary Prevention of Cardiovascular Disease: A Report of the American College of Cardiology/American Heart Association Task Force on Clinical Practice Guidelines. Circulation. 2019;140(11): e596-e646.

42. Fredrickson BL, Cohn MA, Coffey KA, Pek J, Finkel SM. Open hearts build lives: positive emotions, induced through loving-kindness meditation, build consequential personal resources. J Pers Soc Psychol. 2008;95(5):1045-1062.

\section{Figures}




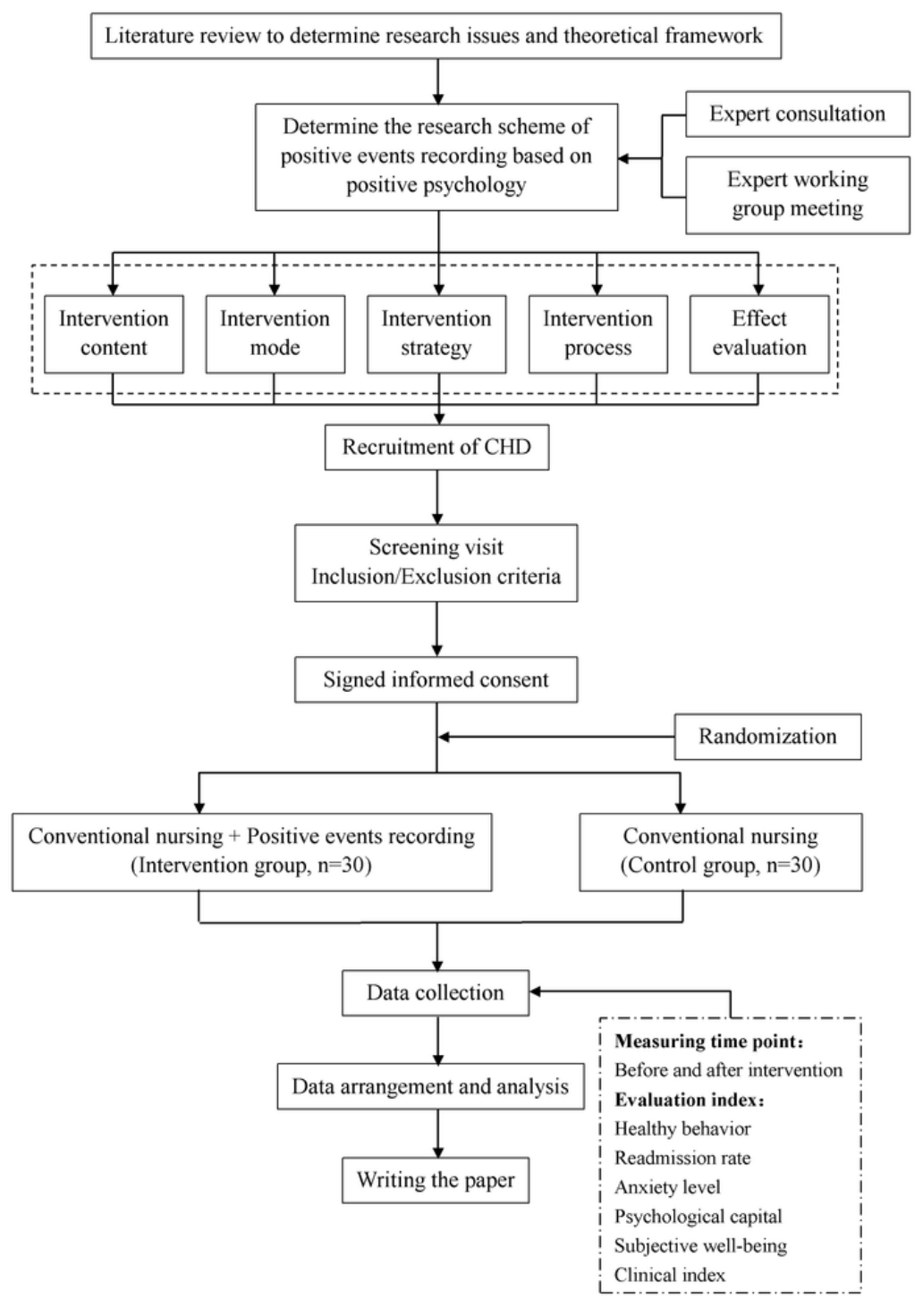

Figure 1

Schematic flow diagram of the study

\section{Supplementary Files}

This is a list of supplementary files associated with this preprint. Click to download.

- ModelconsentformChinese.doc

- SPIRITChecklist2013.doc 